\title{
Consumo e digestibilidade dos nutrientes dietéticos em cordeiros de diferentes grupos genéticos alimentados com farelo de castanha de caju
}

\author{
Intake and digestibility of dietary nutrients in lambs of different genetic groups fed with \\ cashew nut meal
}

\author{
SILVA, Vandenberg Lira' ${ }^{1}$ ROGÉRIO, Marcos Cláudio Pinheiro ${ }^{2}$; BOMFIM, Marco \\ Aurélio Delmondes ${ }^{2}$; LEITE, Eneas Reis ${ }^{3}$; LANDIM, Aline Vieira ${ }^{3}$; ALVES, Arnaud \\ Azevedo ${ }^{4}$; COSTA, Hélio Henrique Araújo ${ }^{1}$; FREIRE, Ana Paula Alves ${ }^{5}$
}

\author{
${ }^{1}$ Universidade Federal de Minas Gerais, Departamento de Zootecnia, Belo Horizonte, Minas Gerais, Brasil. \\ ${ }^{2}$ Embrapa Caprinos e Ovinos, Sobral, Ceará, Brasil. \\ ${ }^{3}$ Universidade Vale do Acaraú, Departamento de Zootecnia, Sobral, Ceará, Brasil. \\ ${ }^{4}$ Universidade Federal do Piauí, Departamento de Zootecnia, Teresina, Piauí, Brasil. \\ ${ }^{5}$ Universidade de São Paulo, Escola Superior de Agricultura Luiz de Queiroz, Departamento de \\ Zootecnia, Piracicaba, São Paulo, Brasil. \\ *Endereço para correspondência: berglira@gmail.com
}

\section{RESUMO}

Objetivou-se avaliar a inclusão do farelo de castanha de caju (FCC) fornecido a cordeiros de três grupos genéticos sobre os consumos e digestibilidades dos nutrientes dietéticos. Foram utilizados dezoito ovinos, machos e inteiros, $1 / 2$ sangue Dorper, $1 / 2$ sangue Somalis e $1 / 2$ sangue Santa Inês, com $22,66 \mathrm{~kg}$ de peso vivo. A dieta controle composta por feno de Aruana, milho, farelo de soja. A dieta teste, composta pelos mesmos alimentos mais o FCC. O delineamento foi o inteiramente casualizado em esquema fatorial $3 \times 2$, três grupos genéticos versus duas dietas (contendo ou não FCC) com três repetições por tratamento. Maiores consumos de nutrientes foram obtidos pelos animais $1 / 2$ sangue Dorper x SRD em relação aos animais 1/2 sangue Santa Inês x SRD, tendo sido ambos semelhantes aos animais $1 / 2$ sangue Somalis $\mathrm{x}$ SRD. Houve interação significativa somente para os coeficientes de digestibilidade da MS. Menores coeficientes de digestibilidade foram observados para os animais $1 / 2$ sangue Santa Inês $x$ SRD nas dietas contendo FCC. Menores digestibilidades da FDA e da HCEL foram observadas nas dietas com FCC. Considerandose os grupos genéticos, maiores digestibilidades da FDN, da FDA e da HCEL foram verificadas para os animais $1 / 2$ sangue Dorper x SRD em comparação aos animais $1 / 2$ sangue Santa Inês $\mathrm{x}$
SRD, sendo ambos genótipos, semelhantes aos animais $1 / 2$ sangue Somalis X SRD. A inclusão de FCC exerce efeito negativo sobre a digestibilidade das frações fibrosas, notadamente sobre a hemicelulose, implicando em redução da digestibilidade da MS dietética nos cordeiros 1/2 sangue Santa Inês.

Palavras-chave: lipídios, nutrição, ovinos, subprodutos

\section{SUMMARY}

This study aimed to evaluate the inclusion of cashew nut meal (CNM) offered to lambs of three genetic groups on intake and digestibility of dietary nutrients. Eighteen male lambs, $1 / 2$ Dorper $x 1 / 2$ native, $1 / 2$ Somalis $x 1 / 2$ native and $1 / 2$ Santa Inês $x 1 / 2$ native, with body weight of 22,66. The control diet, constituted Aruana's hay, corn, soybean meal. The test diet was constituted of these feed plus CNM, Acompletely randomized design in a factorial project $3 \times 2$, three genetic groups versus two diets (with or without CNM) with three replicates per treatment.Higher intakes were obtained for the animals $1 / 2$ Dorper $x 1 / 2$ native compared to animals $1 / 2$ Santa Inês $x 1 / 2$ native, being similar to the animals $1 / 2$ Somalis $x 1 / 2$ 
native. There was significant interaction only for the DM digestibility. Lower digestibilities were observed for animals $1 / 2$ Santa Ines x $1 / 2$ native in the diet containing the CNM. Lower digestibilities of ADF and HCEL were observed in the diets with the inclusion of CNM. For the genetics groups, NDF, ADF and HCEL digestibilities higher it was verified to the animals $1 / 2$ Dorper $x \quad 1 / 2$ native comparedto theanimals $1 / 2$ Santa Inês $x 1 / 2$ native, being both similar to the animals $1 / 2$ Somalis $\mathrm{x} 1 / 2$ native. The inclusion of CNM has a negative effect on the digestibilities of fibrous fractions, especially on the hemicellulose, resulting in reduction on the digestibility of dietary DM in the $1 / 2$ Santa Inês $\mathrm{x} 1 / 2$ native.

Keywords: by-products, lipids, nutrition, sheep

\section{INTRODUÇÃO}

A estacionalidade das chuvas na região Nordeste é um dos principais fatores que interferem na disponibilidade alimentar para os rebanhos, limitando a expressão do potencial genético de pequenos ruminantes (MARQUES et al., 2007). Com a baixa disponibilidade de alimentos e o aumento do preço dos alimentos concentrados utilizados para complementar a deficiência energética e proteica das dietas ingeridas pelos animais no pasto, a utilização de alimentos alternativos disponíveis nessa região torna-se interessante fonte alimentar de baixo custo. Dentre os alimentos, podemos destacar o farelo de castanha de caju, um alimento alternativo com boa disponibilidade nos períodos de estiagem, quando há redução da disponibilidade alimentar de modo geral (PIMENTEL et al., 2007; SILVA et al., 2011), um dos desafios para utilização dos alimentos alternativos são as características físicas e químicas do alimento que podem afetar positivamente ou negativamente a ingestão (YAMAMOTO et al., 2007). Uma das preocupações quanto ao uso do farelo de castanha de caju é sua concentração de lipídios, pois o fornecimento de dietas com mais de $7 \%$ de extrato etéreo para ruminantes podem comprometer a ingestão de nutrientes, bem como implicar em alterações no aproveitamento das frações fibrosas dietéticas no rúmen (MAIA et al., 2006). Além do aspecto nutricional, o potencial genético dos animais deve ser considerado. Devido o domínio dos rebanhos por animais sem padrão racial definido (SPRD), o emprego de cruzamentos desses animais com outros com alto potencial tem sido uma importante ferramenta para a maximização dos indicadores produtivos da exploração de ovinos e consequentemente maior economicidade dos sistemas de produção de ovinos no Nordeste Brasileiro.

A associação das interações envolvendo os diferentes genótipos e o manejo nutrição é de grande importância para o seguimento produtivo, sendo importante usar parâmetros de avaliação como o consumo e a digestibilidade dos nutrientes dietéticos como forma de compreensão da dinâmica de ingestão e a absorção dos nutrientes a partir do trato gastrointestinal (PEREIRA et al., 2008).

Objetivou-se nesse trabalho avaliar a inclusão do farelo de castanha de caju em dietas experimentais fornecidas a ovinos de diferentes grupos genéticos $(1 / 2$ sangue Dorper x SRD, $1 / 2$ sangue Somalis $x$ SRD e $1 / 2$ sangue Santa Inês x SRD), verificando os aspectos de consumo e digestibilidade dos nutrientes dietéticos.

\section{MATERIAL E MÉTODOS}

O experimento foi realizado no setor de digestibilidade animal da Fazenda Experimental Vale do Acaraú (FAEX), 
em uma área pertencente à Universidade Estadual Vale do Acaraú (UVA), em Sobral, Ceará, zona fisiográfica do sertão cearense, em novembro de 2006, sob aprovação do comitê de ética e biosseguridade animal da Universidade. Foram utilizados dezoito cordeiros, machos e inteiros, de três diferentes grupos genéticos $(1 / 2$ sangue Dorper $\mathrm{x}$ SPRD, $1 / 2$ sangue Somalis x SPRD e $1 / 2$ sangue Santa Inês x SPRD) com três meses de idade e peso vivo médio de $22,66 \mathrm{~kg}$ seguindo um delineamento inteiramente casualizado em arranjo fatorial $3 \times 2$ (três grupos genéticos vs duas dietas experimentais, contendo ou não a inclusão de farelo de castanha de caju. As dietas foram formuladas conforme o NRC (1985) para atenderem as exigências nutricionais de cordeiros em terminação com $30 \mathrm{~kg}$ de peso vivo e ganho de peso médio diário de $250 \mathrm{~g} / \mathrm{dia}$. A dieta controle foi composta por feno de capim Aruana (Panicum maximum cv. Aruana), farelo de soja e milho. A esses mesmos ingredientes foi adicionado o farelo de castanha de caju, constituindo a dieta experimental. O maior nível de inclusão do farelo de castanha na dieta foi determinado pelo conteúdo de extrato etéreo, no máximo de $7 \%$ na matéria seca. Os animais foram desmamados aos 89 dias de idade, previamente desverminados e alojados individualmente em gaiolas de metabolismo, alocadas em galpão de alvenaria coberto, com piso concretado, dotadas de comedouros, bebedouros, saleiros plásticos e dispositivos apropriados para coleta de urina e fezes, onde permaneceram durante todo $\mathrm{o}$ período experimental. O ensaio teve duração de 17 dias, sendo 10 dias para a adaptação e sete para coletas. Os animais foram pesados no inicio do período de adaptação, sendo os pesos utilizados para o cálculo do consumo e ajustes na quantidade de sobras diárias.
As dietas foram divididas em duas refeições iguais e fornecidas às oito e às $17 \mathrm{~h}$, buscando-se deixar de 15 a $20 \%$ de matéria seca em relação total fornecido, por dia. Água e mistura mineral estiveram disponíveis à vontade. A composição bromatológica dos alimentos utilizados está apresentada na Tabela 1.

Durante os sete dias de coleta, amostras do alimento oferecido e sobras foram recolhidas diariamente, pesadas e armazenadas em sacos plásticos identificados, em temperatura a $-10^{\circ} \mathrm{C}$. Posteriormente foi preparada uma amostra composta por animal para as sobras dos sete dias de coleta. Após isso, as amostras foram homogeneizadas $\mathrm{e}$ acondicionadas em bandejas de alumínio e levadas à estufa de ventilação forçada $\left(55\right.$ a $60^{\circ} \mathrm{C}$ ) por 72 horas, sendo em seguida moídas em moinho Tomas Myller com peneira com $1 \mathrm{~mm}$ e armazenadas para as análises laboratoriais.

A coleta de fezes foi realizada diariamente. A produção total foi recolhida dos coletores e teve o peso registrado, sendo feita a coleta de uma alíquota de $20 \%$ deste peso, a qual foi embalada em sacos plásticos individuais e guardada em câmara frigorífica a temperatura de $-10^{\circ} \mathrm{C}$. Ao final do experimento, foram descongeladas a temperatura ambiente por 14 horas, sendo preparada uma amostra composta por animal. Após isso, as amostras foram homogeneizadas e acondicionadas em bandejas de alumínio e levadas à estufa de ventilação forçada $\left(55\right.$ a $\left.60^{\circ} \mathrm{C}\right)$ por 72 horas, sendo em seguida moídas em moinho Tomas Myller com peneira com $1 \mathrm{~mm}$ e armazenadas para as análises laboratoriais. As análises laboratoriais foram realizadas nas dependências do Laboratório de Nutrição Animal do curso de Zootecnia do Centro de Ciências Agrárias e Biológicas da UVA. 
Rev. Bras. Saúde Prod. Anim., Salvador, v.14, n.4, p.695-709 out./dez., 2013 http://www.rbspa.ufba.br ISSN 15199940

Tabela 1. Composição bromatológica (\%) dos alimentos

\begin{tabular}{lcccc}
\hline Componentes & $\begin{array}{c}\text { Feno de Capim } \\
\text { Aruana }\end{array}$ & $\begin{array}{c}\text { Farelo de } \\
\text { Castanha }\end{array}$ & Milho & $\begin{array}{c}\text { Farelo de } \\
\text { soja }\end{array}$ \\
\hline Matéria Seca (\%) em base de matéria & 91,25 & 95,53 & 90,7 & 89,56 \\
natural & 7,34 & 24,54 & 10,63 & 45,95 \\
Proteína Bruta (\% na MS) & 57,90 & 54,73 & 93,51 & 31,01 \\
NIDN (\%NT) & 40,87 & 19,87 & 3,53 & 21,35 \\
NIDA (\%NT) & 1,45 & 42,49 & 6,59 & 1,34 \\
Extrato Etéreo (\% na MS) & 78,59 & 21,01 & 18,27 & 19,41 \\
Fibra em Detergente Neutro (\% na & 37,59 & 0,20 & 3,72 & 1,11 \\
MS) & 45,02 & 11,32 & 4,42 & 12,19 \\
FDNVD (\% na MS) & 33,57 & 18,63 & 17,46 & 17,04 \\
Fibra em Detergente Ácido (\% na MS) & 36,73 & 7,84 & 4,03 & 9,8 \\
Hemicelulose (\% na MS) & 4,32 & 3,63 & 0,68 & 1,56 \\
Celulose (\% na MS) & 9,04 & 7,07 & 2,03 & 6,16 \\
Lignina (\% na MS) & 1,2 & 0,1 & 0,9 & 0,27 \\
Cinzas \% na MS) & 0.42 & 0,51 & 0,26 & 0,59 \\
Cálcio & 82,17 & 25,9 & 80,75 & 46,55 \\
Fósforo & 10,52 & 9,77 & 62,86 & 39,95 \\
Carboidratos Totais (\% na MS) & Carboidratos Não Fibrosos (\% na MS) &
\end{tabular}

NIDN = nitrogênio insolúvel em detergente neutro, NIDA = nitrogênio insolúvel em detergente ácido, FDNVD = fibra em detergente neutro verdadeiramente digestível.

Foram realizadas as determinações de matéria seca, matéria mineral, extrato etéreo e proteína bruta do alimento, sobras e fezes seguiram a metodologia proposta pela AOAC (1995). A quantificação da fibra em detergente neutro, fibra em detergente ácido, celulose, hemicelulose e lignina (Van SOEST et al., 1991). Para o cálculo de NDT das dietas experimentais utilizou-se a equação proposta por (SNIFFEN et al., 1992). Para o cálculo da porcentagem dos carboidratos totais (CT), adotou-se a equação sugerida por Sniffen et al. (1992) e para o cálculo dos carboidratos não fibrosos (CNF) utilizou-se equação CNF $=100-(\mathrm{PB}+\mathrm{EE}+\mathrm{MM}+\mathrm{FDN})$. Os coeficientes de digestibilidade de MS, MO, PB, EE, FDN, FDA, CEL, HCEL foram determinados pela diferença entre $\mathrm{O}$ ingerido e excretado nas fezes a partir da seguinte fórmula: [(Consumo do nutriente em gramas - quantidade em gramas do nutriente nas fezes)/Consumo do nutriente em gramas] $]^{*} 100$.

$\mathrm{O}$ delineamento experimental foi $\mathrm{o}$ inteiramente casualizado em esquema fatorial $3 \times 2$, três grupos genéticos e duas dietas experimentais, perfazendo seis tratamentos com três repetições por tratamento. As médias foram comparadas pelo teste SNK em nível de $5 \%$ de probabilidade. As correlações entre as variáveis foram feitas por meio das análises de correlação lineares de Pearson. As análises estatísticas foram realizadas utilizando o software SAEG 8.0. (UFV, 2001) O peso inicial foi utilizado no modelo estatístico como covariável. A composição das dietas experimentais está apresentada na Tabela 2. 
Rev. Bras. Saúde Prod. Anim., Salvador, v.14, n.4, p.695-709 out./dez., 2013 http://www.rbspa.ufba.br ISSN 15199940

Tabela 2. Composição centesimal (\%) e bromatológica (\%) das dietas experimentais, conforme a inclusão ou não de FCC

\begin{tabular}{|c|c|c|c|c|}
\hline \multicolumn{5}{|c|}{ Composição centesimal } \\
\hline Dietas experimentais & Feno de Capim Aruana & FCC & Milho & $\begin{array}{l}\text { Farelo de } \\
\text { Soja }\end{array}$ \\
\hline Com inclusão de FCC & 40,66 & 13,31 & 31,83 & 14,20 \\
\hline Sem inclusão de FCC & 41,47 & - & 39,58 & 18,95 \\
\hline \multicolumn{5}{|c|}{ Composição bromatológica das dietas experimentais } \\
\hline \multicolumn{3}{|l|}{ Componentes } & $\begin{array}{l}\text { Dieta com } \\
\text { FCC }\end{array}$ & $\begin{array}{l}\text { Dieta sem } \\
\text { FCC }\end{array}$ \\
\hline \multicolumn{3}{|c|}{ Matéria Seca em base de matéria natural (\%) } & 91,41 & 90,71 \\
\hline \multicolumn{3}{|c|}{ Proteína Bruta (\% na MS) } & 16,16 & 15,96 \\
\hline \multicolumn{3}{|l|}{ NIDN (\%NT) } & 65,00 & 66,89 \\
\hline \multicolumn{3}{|l|}{ NIDA $(\% \mathrm{NT})$} & 20,58 & 22,39 \\
\hline \multicolumn{3}{|c|}{ Extrato Etéreo (\% na MS) } & 8,53 & 3,46 \\
\hline \multicolumn{3}{|c|}{ Fibra em Detergente Neutro (\% na MS) } & 43,33 & 43,50 \\
\hline \multicolumn{3}{|c|}{$\begin{array}{l}\text { Fibra em Detergente Neutro Verdadeiramente Digestível ( } \% \text { na } \\
\text { MS) }\end{array}$} & 16,66 & 17,27 \\
\hline \multicolumn{3}{|c|}{ Fibra em Detergente Ácido (\% na MS) } & 22,95 & 22,73 \\
\hline \multicolumn{3}{|c|}{ Hemicelulose (\% na MS) } & 24,11 & 24,06 \\
\hline \multicolumn{3}{|l|}{ Celulose (\% na MS) } & 18,65 & 18,68 \\
\hline \multicolumn{3}{|l|}{ Lignina (\% na MS) } & 2,68 & 2,36 \\
\hline \multicolumn{3}{|l|}{ Cinzas } & 6.13 & 5.72 \\
\hline \multicolumn{3}{|c|}{ Carboidratos Totais (\% na MS) } & 69,19 & 74,86 \\
\hline \multicolumn{3}{|l|}{ Cálcio } & 0,83 & 0,91 \\
\hline \multicolumn{3}{|l|}{ Fósforo } & 0,49 & 0,31 \\
\hline \multicolumn{3}{|c|}{ Carboidratos Não Fibrosos (\% na MS) } & 30,83 & 36,24 \\
\hline \multicolumn{3}{|l|}{$\mathrm{NDT}^{*}$} & 79,83 & 75,64 \\
\hline
\end{tabular}

NIDN = nitrogênio insolúvel em detergente neutro, NIDA = nitrogênio insolúvel em detergente ácido, NDT $=$ nutrientes digestíveis totais .

*Conforme Sniffen et al (1992).

\section{RESULTADOS E DISCUSSÃO}

Observa-se que o FCC na dieta teste substituiu o milho e o farelo de soja como previsto. Não houve comprometimento dos níveis de PB e NDT das dietas quando se reduziram as quantidades de milho e farelo de soja (Tabela 1). De modo geral não houve diferenças marcantes entre as dietas experimentais quanto à composição bromatológica. Observou-se que o teor de extrato etéreo foi maior na dieta com FCC e os teores de carboidratos totais, carboidratos não fibrosos e carboidratos não fibrosos verdadeiramente digestíveis foram ligeiramente inferiores aos verificados na dieta controle (sem FCC). Embora as dietas tenham sido formuladas para não ultrapassar o referencial de consumo de $7 \%$ para o valor de extrato etéreo dietético, a seletividade pode ter contribuído para o incremento nos valores de extrato etéreo das dietas efetivamente consumidas (Tabela 2).

Não houve interação significativa $(\mathrm{P}>0,05)$ entre grupos genéticos e dietas para os consumos de matéria seca e matéria orgânica. Houve diferenças significativas quando se compararam os grupos genéticos em termos de CMS digestível (g/UTM). Os dados da pesquisa demonstram que esse parâmetro foi 
Rev. Bras. Saúde Prod. Anim., Salvador, v.14, n.4, p.695-709 out./dez., 2013 http://www.rbspa.ufba.br ISSN 15199940

superior nos animais $1 / 2$ sangue Dorper $\mathrm{x}$ SRD em relação ao verificado nos animais $1 / 2$ sangue Santa Inês x SRD, tendo sido ambos semelhantes aos animais $1 / 2$ sangue Somalis $x$ SRD (Tabela 3).

De acordo com o NRC (2007), considerando-se a exigência nutricional de cordeiros com maturidade tardia, o valor referencial de consumo de $62,39 \mathrm{~g}$ de MS/UTM/dia assemelha-se mais ao verificado para animais $1 / 2$ sangue Santa
Inês $x$ SRD $(68,92 \mathrm{~g} / \mathrm{UTM})$. Ao considerarmos os dados do referido sistema para cordeiros com maturidade precoce, o valor é $87,76 \mathrm{~g}$ de MS/UTM. Considerando os grupos genéticos, os animais $1 / 2$ sangue Dorper $x \quad$ SRD apresentaram valor semelhante a este $(83,11 \mathrm{~g} / \mathrm{UTM})$. Entre os dois, situa-se o consumo pelos animais $1 / 2$ sangue Somalis x SRD $(78,56 \mathrm{~g} / \mathrm{UTM})$, mais próximo numericamente ao valor para cordeiros de maturidade precoce.

Tabela 3. Valores médios de consumo de nutrientes por ovinos de diferentes genótipos recebendo dietas contendo ou não farelo de castanha de caju (FCC)

\begin{tabular}{|c|c|c|c|}
\hline \multirow{2}{*}{ Grupo Genético } & \multicolumn{2}{|c|}{ CMS } & \multirow{2}{*}{ CMS Dig } \\
\hline & g/UTM & $\% \mathrm{PV}$ & \\
\hline $1 / 2$ sangue Dorper $x$ SRD & $83,11^{\mathrm{a}}$ & $3,68^{\mathrm{a}}$ & $61,88^{\mathrm{a}}$ \\
\hline $1 / 2$ sangue Somalis x SRD & $78,56^{\mathrm{a}}$ & $3,70^{\mathrm{a}}$ & $55,94^{\mathrm{ab}}$ \\
\hline 1/2 sangue Santa Inês x SRD & $68,92^{\mathrm{a}}$ & $3,42^{\mathrm{a}}$ & $48,26^{\mathrm{b}}$ \\
\hline \multirow{2}{*}{ Dietas } & \multicolumn{2}{|c|}{ CMS } & \multirow{2}{*}{ CMS Dig } \\
\hline & g/UTM & $\% \mathrm{PV}$ & \\
\hline Com FCC & $75,12^{\mathrm{a}}$ & $3,59^{\mathrm{a}}$ & $53,43^{\mathrm{a}}$ \\
\hline Sem FCC & $78,61^{\mathrm{a}}$ & $3,60^{\mathrm{a}}$ & $57,29^{\mathrm{a}}$ \\
\hline $\mathrm{CV}(\%)$ & $13,20 \%$ & $13,11 \%$ & $14,40 \%$ \\
\hline \multirow{2}{*}{ Grupo Genético } & \multicolumn{2}{|c|}{$\mathrm{CMO}$} & \multirow{2}{*}{ CMO Dig } \\
\hline & g/UTM & $\% \mathrm{PV}$ & \\
\hline $1 / 2$ sangue Dorper $x$ SRD & $71,29^{\mathrm{a}}$ & $3,16^{\mathrm{a}}$ & $55,44^{\mathrm{a}}$ \\
\hline $1 / 2$ sangue Somalis $x$ SRD & $67,08^{\mathrm{a}}$ & $3,15^{\mathrm{a}}$ & $50,15^{\mathrm{a}}$ \\
\hline 1/2 sangue Santa Inês x SRD & $58,91^{\mathrm{a}}$ & $2,92^{\mathrm{a}}$ & $44,33^{\mathrm{a}}$ \\
\hline \multirow{2}{*}{ Dietas } & \multicolumn{2}{|c|}{$\mathrm{CMO}$} & \multirow{2}{*}{ CMO Dig } \\
\hline & g/UTM & $\% \mathrm{PV}$ & \\
\hline Com FCC & $64,49^{\mathrm{a}}$ & $3,09^{\mathrm{a}}$ & $48,70^{\mathrm{a}}$ \\
\hline Sem FCC & $67,03^{\mathrm{a}}$ & $3,07^{\mathrm{a}}$ & $51,25^{\mathrm{a}}$ \\
\hline $\mathrm{CV}(\%)$ & $13,63 \%$ & $13,48 \%$ & $14,61 \%$ \\
\hline
\end{tabular}

*Médias com letras minúsculas iguais na mesma coluna não diferem significativamente pelo teste SNK $(\mathrm{P}>0,05)$.

CMS = consumo de matéria seca, $\mathrm{CMO}=$ consumo de matéria orgânica, $\mathrm{PV}=$ peso vivo, $\mathrm{UTM}=$ unidade de tamanho metabólico.

$\mathrm{Na}$ atual pesquisa, as diferenças de ingestão de nutrientes entre os genótipos podem ser reflexo das alterações na capacidade do trato digestivo entre os grupos genéticos em questão, o que possivelmente possibilitou uma maior ingestão de nutrientes pelos animais $1 / 2$ sangue Dorper x SPRD em relação aos animais $1 / 2$ sangue Santa Inês. Ressalta-se também o efeito fisiológico da regulação 
do consumo em ruminantes. Nesse contexto, as demandas necessárias ao potencial de desempenho dos animais $1 / 2$ sangue Santa Inês podem ter sido alcançadas mais facilmente em relação aos animais $1 / 2$ sangue Dorper $x$ SRD. Essa condição fisiológica é alcançada quando a absorção de nutrientes proteicos e energéticos atendem os requisitos nutricionais da categoria. No presente estudo, as rações formuladas para os diferentes genótipos estudados tiveram 59,35\% (sem o FCC) e 59,57\% (com o FCC) da fração concentrada, o que pode ter contribuido para limitar o consumo de matéria orgânica, especialmente em cordeiros mestiços Santa Inês $x$ SPRD. No estudo conduzido por Barros et al. (2003) com animais $1 / 2$ sangue Somalis e $1 / 2$ sangue Santa Inês com peso vivo médio de $14,65 \mathrm{~kg}$ alimentados com níveis crescentes de concentrado composto de milho, farelo de soja e sal mineral, os autores verificaram que quantidades de concentrado maiores do que $35,3 \%$ de matéria seca diminuíram o consumo de matéria orgânica nas dietas fornecidas para os cordeiros mestiços Somalis $\mathrm{x}$ SPRD e Santa Inês $x$ SPRD. Os resultados para CMS (g/UTM), foram superiores aos obtidos por Rodrigues et al. (2003), que obtiveram consumos médios de MS 70,54g/UTM, quando avaliaram a inclusão de FCC em níveis de inclusão na fração concentrada de 0 , 12,24 e $36 \%$ em dietas para ovinos. Ao estudar o consumo de nutrientes e aspectos quantitativos da carcaça de cordeiros em terminação recebendo dietas contendo farelo de castanha de caju, Nascimento et al. (2012) observaram valores médios de consumo de MS de 74,3g/UTM e consumo de MO de 64,36g/UTM, semelhantes aos obtidos nesta pesquisa.

Não houve interação $(\mathrm{P}>0,05)$ entre grupos genéticos e dietas experimentais para os consumos de $\mathrm{PB}$ e de EE. Não houve diferença significativa considerando-se as duas dietas experimentais para os consumos de PB e de EE (Tabela 4). Em termos de consumo de $\mathrm{PB}$, houve diferenças para o consumo de proteína digestível para os animais $1 / 2$ sangue Santa Inês x SRD em comparação aos animais $1 / 2$ sangue Dorper $x$ SRD, sendo ambos semelhantes aos animais $1 / 2$ sangue Somalis $x$ SRD. Para o consumo de $E E$, não houve diferença significativa $(\mathrm{P}>0,05)$ para os grupos genéticos, porém, constataram-se maiores consumos nas dietas com FCC $(\mathrm{P}<0,05)$, reflexo do conteúdo mais alto de EE nessas dietas (Tabela 2). Pela correlação de Pearson, percebe-se maior influência do consumo de PB sobre o consumo de MS $(\mathrm{r}=0,9817 ; \quad \mathrm{P}<0,001)$ do que do consumo de EE sobre o consumo de MS $(\mathrm{r}=0,2396 ; \mathrm{P}>0,05)$. Portanto, embora Maia et al. (2006) tenha recomendado que o limite de EE não deve ultrapassar $7 \%$ do total dietético, não houve efeito do consumo de EE sobre o consumo de MS.

O consumo diário de $\mathrm{PB}$ citado pelo NRC (2007) para cordeiros com maturidade tardia deve ficar entre 11,21 e $12,27 \mathrm{~g}$ de PB/UTM. Para cordeiros com maturidade precoce, deve ser entre 10,26 e $11,21 \mathrm{~g}$ de PB/UTM. Os valores médios apresentados na Tabela 4 foram superiores a esses referenciais. De acordo com esse dado, o fornecimento proteico pode ter sido excessivo para todos os grupos genéticos estudados, notadamente para os animais $1 / 2$ sangue Dorper x SRD. Os resultados obtidos nessa pesquisa foram superiores aos observados por Rodrigues et al. (2003) ao avaliarem a inclusão de FCC em níveis de $0,12,24$ e $36 \%$ em dietas para ovinos, que obtiveram valores de consumos médios de PB da ordem de $12,15 \mathrm{~g} / \mathrm{UTM}$. 
Rev. Bras. Saúde Prod. Anim., Salvador, v.14, n.4, p.695-709 out./dez., 2013 http://www.rbspa.ufba.br ISSN 15199940

Tabela 4. Valores médios de consumo de nutrientes por ovinos de diferentes genótipos recebendo dietas contendo ou não farelo de castanha de caju (FCC)

\begin{tabular}{|c|c|c|c|}
\hline \multirow{2}{*}{ Grupo Genético } & \multicolumn{2}{|c|}{ CPB } & \multirow{2}{*}{ CPB Dig } \\
\hline & g/UTM & $\% \mathrm{PV}$ & \\
\hline $1 / 2$ sangue Dorper $x$ SRD & $16,14^{\mathrm{a}}$ & $0,72^{\mathrm{a}}$ & $12,61^{\mathrm{a}}$ \\
\hline $1 / 2$ sangue Somalis $x$ SRD & $15,50^{\mathrm{a}}$ & $0,73^{\mathrm{a}}$ & $11,83^{\mathrm{ab}}$ \\
\hline 1/2 sangue Santa Inês x SRD & $13,72^{\mathrm{a}}$ & $0,68^{\mathrm{a}}$ & $10,49^{\mathrm{b}}$ \\
\hline \multirow{2}{*}{ Dietas } & \multicolumn{2}{|c|}{ CPB } & \multirow{2}{*}{ CPB Dig } \\
\hline & g/UTM & $\% \mathrm{PV}$ & \\
\hline Com FCC & $14,84^{\mathrm{a}}$ & $0,70^{\mathrm{a}}$ & $11,74^{\mathrm{a}}$ \\
\hline Sem FCC & $15,39^{\mathrm{a}}$ & $0,71^{\mathrm{a}}$ & $11,75^{\mathrm{a}}$ \\
\hline $\mathrm{CV}(\%)$ & $12,86 \%$ & $12,99 \%$ & $10,34 \%$ \\
\hline \multirow{2}{*}{ Grupo Genético } & \multicolumn{2}{|c|}{ CEE } & \multirow{2}{*}{ CEE Dig } \\
\hline & g/UTM & $\% \mathrm{PV}$ & \\
\hline $1 / 2$ sangue Dorper $x$ SRD & $6,02^{\mathrm{a}}$ & $0,27^{\mathrm{a}}$ & $5,53^{\mathrm{a}}$ \\
\hline $1 / 2$ sangue Somalis $x$ SRD & $5,69^{\mathrm{a}}$ & $0,27^{\mathrm{a}}$ & $5,14^{\mathrm{a}}$ \\
\hline 1/2 sangue Santa Inês x SRD & $4,82^{\mathrm{a}}$ & $0,25^{\mathrm{a}}$ & $4,44^{\mathrm{a}}$ \\
\hline \multirow{2}{*}{ Dietas } & \multicolumn{2}{|c|}{ CEE } & \multirow{2}{*}{ CEE Dig } \\
\hline & g/UTM & $\% \mathrm{PV}$ & \\
\hline Com FCC & $7,71^{\mathrm{a}}$ & $0,37^{\mathrm{a}}$ & $7,20^{\mathrm{a}}$ \\
\hline Sem FCC & $3,32^{\mathrm{b}}$ & $0,15^{\mathrm{b}}$ & $2,87^{\mathrm{b}}$ \\
\hline $\mathrm{CV}(\%)$ & $14,67 \%$ & $15,23 \%$ & $16,31 \%$ \\
\hline
\end{tabular}

*Médias com letras minúsculas iguais na mesma coluna não diferem significativamente pelo teste SNK $(\mathrm{P}>0,05)$.

$\mathrm{CPB}=$ consumo de proteína bruta, $\mathrm{CEE}=$ consumo de extrato etéreo, $\mathrm{PV}=$ peso vivo, $\mathrm{UTM}=$ unidade de tamanho metabólico.

Não houve interação (P>0,05) entre grupos genéticos e dietas para $\mathrm{O}$ consumo das frações fibrosas e seus constituintes digestíveis (Tabela 5). Não houve efeito das dietas sobre os parâmetros citados, assim como não houve efeito dos grupos genéticos estudados sobre o consumo de celulose. Houve diferenças efeito do grupo genético sobre os consumos de FDN, FDA e HCEL (g/UTM), bem como para os consumos de FDN, FDA e HCEL digestíveis. Verificaram-se que os animais $1 / 2$ sangue Dorper $x$ SRD em relação aos animais $1 / 2$ sangue Santa Inês x SRD, tendo sido o consumo dos animais $1 / 2$ sangue Somalis $x$ SRD semelhante a ambos. Um estudo realizado por Macedo Júnior et al. (2006) determinou que para ovelhas no final de gestação, há a necessidade de no mínimo $28,05 \%$ de FDN na composição dietética para manutenção da função ruminal normal, correspondendo assim a um consumo de $78,18 \mathrm{~g} / \mathrm{kg}^{0,75}$. Considerando-se esse valor em relação aos consumos de MS recomendados pelo NRC (2007) para cordeiros de maturidade tardia $(62,39 \mathrm{~g} / \mathrm{UTM})$ e para cordeiros de maturidade precoce $(87,76 \mathrm{~g} / \mathrm{UTM})$ obtêm-se os valores mínimos de 17,52g de FDN/UTM para cordeiros de maturidade tardia e 24,64g de FDN/UTM para cordeiros de maturidade precoce. Portanto, para todos os grupamentos genéticos estudados, os valores mínimos foram atendidos conforme essa recomendação. 
Rev. Bras. Saúde Prod. Anim., Salvador, v.14, n.4, p.695-709 out./dez., 2013 http://www.rbspa.ufba.br ISSN 15199940

Tabela 5. Valores médios de consumo de frações fibrosas por ovinos de diferentes genótipos recebendo dietas contendo ou não farelo de castanha de caju (FCC)

\begin{tabular}{|c|c|c|c|}
\hline \multirow{2}{*}{$\begin{array}{l}\text { Grupo } \\
\text { Genético }\end{array}$} & \multicolumn{2}{|c|}{ CFDN } & \multirow{2}{*}{$\begin{array}{c}\text { CFDN } \\
\text { Dig }\end{array}$} \\
\hline & $\mathrm{g} / \mathrm{UTM}$ & $\% \mathrm{PV}$ & \\
\hline $\begin{array}{l}1 / 2 \text { sangue } \\
\text { Dorper } x \\
\text { SRD }\end{array}$ & $33,14^{\mathrm{a}}$ & $1,49^{\mathrm{a}}$ & $18,46^{\mathrm{a}}$ \\
\hline $\begin{array}{l}1 / 2 \text { sangue } \\
\text { Somalis } x \\
\text { SRD }\end{array}$ & $30,35^{\mathrm{ab}}$ & $1,43^{\mathrm{a}}$ & $16,00^{\mathrm{ab}}$ \\
\hline $\begin{array}{l}1 / 2 \text { sangue } \\
\text { Santa Inês } \\
x \text { SRD }\end{array}$ & $25,44^{\mathrm{b}}$ & $1,24^{\mathrm{a}}$ & $12,66^{\mathrm{b}}$ \\
\hline \multirow{2}{*}{ Dietas } & \multicolumn{2}{|c|}{ CFDN } & CFDN \\
\hline & g/UTM & $\% \mathrm{PV}$ & Dig \\
\hline Com FCC & $28,70^{\mathrm{a}}$ & $1,37^{\mathrm{a}}$ & $14,58^{\mathrm{a}}$ \\
\hline Sem FCC & $30,58^{\mathrm{a}}$ & $1,40^{\mathrm{a}}$ & $16,84^{\mathrm{a}}$ \\
\hline $\mathrm{CV}(\%)$ & $14,47 \%$ & $14,50 \%$ & $20,61 \%$ \\
\hline \multirow{2}{*}{$\begin{array}{l}\text { Grupo } \\
\text { Genético }\end{array}$} & \multicolumn{2}{|c|}{ CFDA } & CFDA \\
\hline & g/UTM & $\% \mathrm{PV}$ & Dig \\
\hline $\begin{array}{l}1 / 2 \text { sangue } \\
\text { Dorper } x \\
\text { SRD } \\
1 / 2 \text { sangue }\end{array}$ & $16,67^{\mathrm{a}}$ & $0,75^{\mathrm{a}}$ & $7,79^{\mathrm{a}}$ \\
\hline $\begin{array}{l}\text { Somalis } \mathrm{x} \\
\text { SRD }\end{array}$ & $15,69^{\mathrm{ab}}$ & $0,74^{\mathrm{a}}$ & $7,00^{\mathrm{ab}}$ \\
\hline $\begin{array}{l}1 / 2 \text { sangue } \\
\text { Santa Inês } \\
\text { x SRD }\end{array}$ & $12,98^{\mathrm{b}}$ & $0,63^{\mathrm{a}}$ & $5,68^{\mathrm{b}}$ \\
\hline \multirow{2}{*}{ Dietas } & \multicolumn{2}{|c|}{ CFDA } & CFDA \\
\hline & $\mathrm{g} / \mathrm{UTM}$ & $\% \mathrm{PV}$ & Dig \\
\hline Com FCC & $14,76^{\mathrm{a}}$ & $0,71^{\mathrm{a}}$ & $6,49^{\mathrm{a}}$ \\
\hline Sem FCC & $15,48^{\mathrm{a}}$ & $0,71^{\mathrm{a}}$ & $7,15^{\mathrm{a}}$ \\
\hline $\mathrm{CV}(\%)$ & $14,58 \%$ & $15,54 \%$ & $19,02 \%$ \\
\hline \multirow{2}{*}{$\begin{array}{l}\text { Grupo } \\
\text { Genético }\end{array}$} & \multicolumn{2}{|c|}{ CHCEL } & CHCEL \\
\hline & $\mathrm{g} / \mathrm{UTM}$ & $\% \mathrm{PV}$ & Dig \\
\hline $\begin{array}{l}1 / 2 \text { sangue } \\
\text { Dorper } x \\
\text { SRD } \\
1 / 2 \text { sanoue }\end{array}$ & $18,58^{\mathrm{a}}$ & $0,84^{\mathrm{a}}$ & $12,60^{\mathrm{a}}$ \\
\hline $\begin{array}{l}\text { Somalis } x \\
\text { SRD }\end{array}$ & $17,01^{\mathrm{ab}}$ & $0,80^{\mathrm{a}}$ & $11,17^{\mathrm{ab}}$ \\
\hline $\begin{array}{l}1 / 2 \text { sangue } \\
\text { Santa Inês } \\
\text { x SRD }\end{array}$ & $14,15^{\mathrm{b}}$ & $0,69^{\mathrm{a}}$ & $9,13^{\mathrm{b}}$ \\
\hline \multirow{2}{*}{ Dietas } & \multicolumn{2}{|c|}{ CHCEL } & CHCEL \\
\hline & g/UTM & $\% \mathrm{PV}$ & Dig \\
\hline
\end{tabular}

\begin{tabular}{|c|c|c|c|}
\hline Com FCC & $16,04^{\mathrm{a}}$ & $0,77^{\mathrm{a}}$ & $10,33^{\mathrm{a}}$ \\
\hline Sem FCC & $17,13^{\mathrm{a}}$ & $0,78^{\mathrm{a}}$ & $11,60^{\mathrm{a}}$ \\
\hline $\mathrm{CV}(\%)$ & $13,83 \%$ & $14,11 \%$ & $18,16 \%$ \\
\hline Grupo & \multicolumn{2}{|c|}{ CCEL } & CCEL \\
\hline Genético & $\mathrm{g} / \mathrm{UTM}$ & $\% \mathrm{PV}$ & Dig \\
\hline $\begin{array}{l}1 / 2 \text { sangue } \\
\text { Dorper } x \\
\text { SRD }\end{array}$ & $13,81^{\mathrm{a}}$ & $0,62^{\mathrm{a}}$ & $6,03^{\mathrm{a}}$ \\
\hline $\begin{array}{l}1 / 2 \text { sangue } \\
\text { Somalis } x \\
\text { SRD }\end{array}$ & $12,89^{\mathrm{a}}$ & $0,61^{\mathrm{a}}$ & $5,49^{\mathrm{a}}$ \\
\hline $\begin{array}{l}1 / 2 \text { sangue } \\
\text { Santa Inês } \\
\text { x SRD }\end{array}$ & $10,85^{\mathrm{a}}$ & $0,53^{\mathrm{a}}$ & $4,44^{\mathrm{a}}$ \\
\hline \multirow{2}{*}{ Dietas } & \multicolumn{2}{|c|}{ CCEL } & CCEL \\
\hline & g/UTM & $\% \mathrm{PV}$ & Dig \\
\hline Com FCC & $12,39^{\mathrm{a}}$ & $0,59^{\mathrm{a}}$ & $4,98^{\mathrm{a}}$ \\
\hline Sem FCC & $12,65^{\mathrm{a}}$ & $0,58^{\mathrm{a}}$ & $5,66^{\mathrm{a}}$ \\
\hline $\mathrm{CV}(\%)$ & $18,67 \%$ & $15,19 \%$ & $30,37 \%$ \\
\hline
\end{tabular}

Médias com letras minúsculas iguais na mesma coluna não diferem significativamente pelo teste $\mathrm{SNK}(\mathrm{P}>0,05)$

CFDN = consumo de fibra em detergente neutro, $\mathrm{CFDA}=$ consumo de fibra $\mathrm{em}$ detergente ácido, CHCEL = consumo de hemicelulose, $\mathrm{CCEl}=$ consumo de celulose, $\mathrm{PV}$ $=$ peso vivo, UTM = unidade de tamanho metabólico. 
O consumo de MS (g/UTM) foi influenciado pelo consumo de FDN (g/UTM) $\quad(\mathrm{r}=0,9162 ; \quad \mathrm{P}<0,0001)$, tornando-se importante destacar que a proporção de consumo de FDN $(43,33$ e $43,50 \%)$ e de FDA (22,95 e 22,73\%) em relação ao consumo de MS nas dietas experimentais foram baixos. Esse dado corrobora com os comentários de que o efeito da dieta sobre os grupos genéticos teve um efeito mais marcante sobre o consumo de MS por causa dos níveis energéticos dietéticos do que propriamente do efeito da fibra sobre a capacidade de enchimento do rúmen. Pode-se constatar, pela análise das dietas experimentais (Tabela 2), que a maior fração da FDN é composta por celulose e hemicelulose, não sendo verificados efeitos marcantes da lignificação da parede celular sobre o consumo. O valor de lignina das dietas experimentais foram baixos (Tabela 2). Quando a fibra é incluída em excesso na dieta, sua densidade energética torna-se baixa, seu consumo é reduzido, e a produtividade animal tende a diminuir.
No entanto, quando níveis mínimos de fibra não são atendidos, ou ainda, quando o tamanho de partículas da forragem é inadequado, há o comprometimento da produção de saliva, redução do $\mathrm{pH}$ ruminal e redução da ruminação (LOPES et al., 2008).

Houve interação significativa entre grupos genéticos e dietas experimentais $(\mathrm{P}<0,05)$ para os coeficientes de digestibilidade da MS (Tabela 6). A digestibilidade da MS pelos animais $1 / 2$ sangue Santa Inês x SRD foi a menor dentre os grupos genéticos avaliados, nas dietas que incluíram FCC, enquanto não houve diferença entre os grupos genéticos para dieta controle. Apenas dentro do grupo $1 / 2$ sangue Santa Inês $x$ SRD houve diferenças para a digestibilidade da MS, considerando-se as dietas controle e teste, onde observou-se para os animais $1 / 2$ sangue Santa Inês x SRD que receberam a dieta com FCC, menor digestibilidade da MS $(66,23 \%)$ em relação aos animais do mesmo grupo que receberam a dieta sem a inclusão de FCC $(73,20 \%)$.

Tabela 6. Média dos coeficientes de digestibilidade da matéria seca de dietas contendo ou não farelo de castanha por ovinos de diferentes grupos genéticos

\begin{tabular}{lcccc}
\hline & \multicolumn{4}{c}{ Digestibilidade da Matéria Seca $(\%)(\mathrm{CV}=3,02 \%)$} \\
\cline { 2 - 4 } Inclusão de FCC & \multicolumn{3}{c}{ Grupos Genéticos } \\
\cline { 2 - 4 } & Dorper x SRD & Somalis x SRD & Santa Inês x SRD & \\
\hline Com Inclusão & $74,05^{\mathrm{Aa}}$ & $71,51^{\mathrm{Aa}}$ & $66,23^{\mathrm{Bb}}$ & $70,60^{\mathrm{A}}$ \\
Sem Inclusão & $74,66^{\mathrm{Aa}}$ & $70,66^{\mathrm{Aa}}$ & $73,20^{\mathrm{Aa}}$ & $72,84^{\mathrm{A}}$ \\
\hline Médias & $74,36^{\mathrm{a}}$ & $71,08^{\mathrm{b}}$ & $69,71^{\mathrm{b}}$ & \\
\hline
\end{tabular}

${ }^{\mathrm{a}}$ Médias com letras minúsculas iguais na mesma linha indicam semelhança estatística (P>0,05).

${ }^{\mathrm{A}}$ Médias com letras maiúsculas iguais na mesma coluna indicam semelhança estatística $(\mathrm{P}>0,05)$.

Não houve interação significativa $(\mathrm{P}>0,05)$ para as digestibilidades da MO, PB e EE (Tabela 7). Também não foram observadas diferenças significativas para as digestibilidades da MO e PB, considerando-se as dietas experimentais e os grupos genéticos. Houve diferenças para a digestibilidade do EE considerando-se as dietas, sendo observadas maiores digestibilidades desse nutriente para as dietas contendo FCC. Embora tenha havido diferenças em 
termos de consumo de MS e PB digestível (Tabelas 3 e 4), não houve diferenças entre as digestibilidades de MS e PB (Tabelas 6 e 7). Os herbívoros, de modo geral, aumentam o consumo na tentativa de vencer a baixa digestibilidade do alimento reduzindo o tempo de retenção (Van SOEST, 1994). As diferenças de digestibilidade do EE ocorreram basicamente pelo efeito dietético (Tabela 7). A maior inclusão do FCC pode ter favorecido $\mathrm{O}$ incremento da digestibilidade desse nutriente nas dietas que incluíram o FCC. Essa informação corrobora com os resultados verificados por Pimentel et al. (2011) em que observaram aumento linear da digestibilidade de EE com o aumento da inclusão de farelo de castanha, rico em lipídios, em dietas fornecidas para ovinos. Não houve efeito marcante das digestibilidades da PB ( $r=0,4997 ; \mathrm{P}>0,05)$ e do EE ( $r=-$ $0,1595 ; \mathrm{P}>0,05)$ sobre a digestibilidade da MS. O consumo e a digestibilidade das frações fibrosas devem ter exercido efeito mais representativo sobre a digestibilidade da MS e discutidos anteriormente. A digestibilidade da MS, $71,72 \%$, superior à obtida por Cunha et al. (2008) para dietas contendo até $40 \%$ de caroço de algodão integral para ovinos Santa Inês. Digestibilidade da MS inferior foram citados Santos et al. (2009), ao avaliar ovinos Santa Inês alimentados com canola em grão integral, farelo de canola e torta de canola, de 67,33, 66,48 e 66,43\%, respectivamente.

Tabela 7. Média dos coeficientes de digestibilidade da matéria orgânica (DMO), proteína bruta (DPB) e extrato etéreo (DEE) por ovinos de diferentes grupos genéticos recebendo dietas contendo ou não farelo de castanha

\begin{tabular}{lccc}
\hline Grupo Genético & $\begin{array}{c}\text { Digestibilidade } \\
(\mathrm{MO})\end{array}$ & $\begin{array}{c}\text { Digestibilidade } \\
(\mathrm{PB})\end{array}$ & $\begin{array}{c}\text { Digestibilidade do } \\
(\mathrm{EE})\end{array}$ \\
\hline 1/2 sangue Dorper x SRD & $77,70^{\mathrm{a}}$ & $78,44^{\mathrm{a}}$ & $89,83^{\mathrm{a}}$ \\
1/2 sangue Somalis x SRD & $74,65^{\mathrm{a}}$ & $76,33^{\mathrm{a}}$ & $89,20^{\mathrm{a}}$ \\
1/2 sangue Santa Inês x SRD & $75,06^{\mathrm{a}}$ & $76,51^{\mathrm{a}}$ & $90,98^{\mathrm{a}}$ \\
\hline \multirow{2}{*}{ Dietas } & Digestibilidade & Digestibilidade & Digestibilidade do \\
& $(\mathrm{MO})$ & $(\mathrm{PB})$ & $(\mathrm{EE})$ \\
\hline Com FCC & $75,18^{\mathrm{a}}$ & $77,89^{\mathrm{a}}$ & $93,44^{\mathrm{a}}$ \\
Sem FCC & $76,42^{\mathrm{a}}$ & $76,59^{\mathrm{a}}$ & $86,57^{\mathrm{b}}$ \\
\hline CV $(\%)$ & $2,94 \%$ & $3,69 \%$ & $3,19 \%$ \\
\hline
\end{tabular}

*Médias com letras minúsculas iguais na mesma coluna não diferem pelo teste SNK (P>0,05).

Não houve interação $(\mathrm{P}>0,05)$ para a digestibilidade das frações fibrosas (Tabela 8). Considerando-se a digestibilidade da FDN e HCEL, houve diferenças entre as dietas experimentais, onde maiores digestibilidades foram observadas para as dietas em que não houve a inclusão de FCC, não sendo constatadas diferenças para as digestibilidades da FDA e da celulose considerando-se as duas dietas experimentais. Houve efeito do grupo genético sobre a digestibilidade de FDN, FDA e HCEL, sendo observado para os animais $1 / 2$ sangue Santa Inês $x$ SRD valores inferiores aos verificados para os animais $1 / 2$ sangue Dorper $\mathrm{X}$ $\mathrm{SRD}$, sendo ambos semelhantes àqueles dos animais $1 / 2$ sangue Somalis x SRD. 
Observa-se, portanto, um efeito marcante da inclusão do FCC sobre a digestibilidade da FDN, notadamente sobre a hemicelulose. De acordo com Van Soest (1994), ocorre uma variação no grau de associação da fibra (predominantemente celulose) com a digestibilidade. A partir dos dados experimentais, percebe-se que a fração lignocelulose que compõe a FDA parece não ter exercido efeito marcante sobre a digestibilidade da FDN, já que não houve variação da digestibilidade da FDA entre as dietas. Esse fato se comprova na medida em que a correlação entre digestibilidade da hemicelulose e da FDN ( $\mathrm{r}=0,8043$; $\mathrm{P}<0,0001)$ foi maior que a correlação existente entre a digestibilidade da FDN e da celulose $(\mathrm{r}=0,6833 ; \mathrm{P}<0,001)$. O efeito da lignina das dietas experimentais $(2,68 \%$ na MS para dieta com FCC e 2,36\% na MS para dieta sem FCC, Tabela 2), provavelmente não exerceu efeitos deletérios sobre a dieta com FCC.

Tabela 8. Média do coeficiente de digestibilidade das frações fibrosas de dietas contendo ou não farelo de castanha por ovinos de grupos genéticos distintos

\begin{tabular}{llccc}
\hline Grupo Genético & DFDN & DFDA & DHCEL & CEL \\
\hline $1 / 2$ sangue Dorper x SRD & $55,21^{\mathrm{a}}$ & $47,61^{\mathrm{a}}$ & $68,67^{\mathrm{a}}$ & $44,69^{\mathrm{a}}$ \\
$1 / 2$ sangue Somalis x SRD & $52,53^{\mathrm{ab}}$ & $44,47^{\mathrm{ab}}$ & $65,50^{\mathrm{ab}}$ & $42,09^{\mathrm{a}}$ \\
$1 / 2$ sangue Santa Inês x SRD & $49,34^{\mathrm{b}}$ & $42,54^{\mathrm{b}}$ & $62,81^{\mathrm{b}}$ & $38,73^{\mathrm{a}}$ \\
\hline Dietas & DFDN & DFDA & DHCEL & DCEL \\
\hline Com FCC & $50,04^{\mathrm{b}}$ & $43,59^{\mathrm{a}}$ & $63,64^{\mathrm{b}}$ & $39,30^{\mathrm{a}}$ \\
Sem FCC & $54,68^{\mathrm{a}}$ & $46,16^{\mathrm{a}}$ & $67,68^{\mathrm{a}}$ & $44,37^{\mathrm{a}}$ \\
\hline CV $(\%)$ & $6,99 \%$ & $6,48 \%$ & $5,42 \%$ & $18,67 \%$ \\
\hline
\end{tabular}

Médias com letras minúsculas iguais na mesma coluna não diferem significativamente pelo teste SNK $(\mathrm{P}>0,05)$.

DFDN = digestibilidade da fibra em detergente neutro, DFDA = digestibilidade da fibra em detergente ácido, DHCEL = digestibilidade da hemicelulose, $\mathrm{DCEL}=$ digestibilidade da celulose .

Segundo Teixeira \& Borges (2005) as oleaginosas são as fontes de lipídios mais usadas na dieta de ruminantes por proporcionarem alta densidade energética em substituição aos carboidratos rapidamente fermentáveis, favorecendo a fermentação ruminal e a digestão da fibra, entretanto, não deve ser usado em excesso, devido ao seu conteúdo em óleo (SILVA et al., 2007). O elevado teor de extrato etéreo, superior a $7 \%$, pode comprometer a ação dos microrganismos sobre a degradação da fibra dietética. Nesse estudo, a dieta contendo a inclusão de FCC, por sua constituição lipídica mais elevada que a dieta controle (Tabela 2), pode ter prejudicado o processo de degradação ruminal da fibra, particularmente sobre a hemicelulose. As diferenças de digestibilidade da fibra entre os grupos genéticos parecem indicar uma maior sensibilidade à inclusão dietética do FCC nos animais $1 / 2$ sangue Santa Inês em relação aos animais $1 / 2$ sangue Dorper. Esses resultados corroboram com os observados por Rodrigues et al. (2003) trabalhando diferentes níveis de inclusão de FCC (0 a 36\%) na terminação de ovinos em confinamento. Os autores verificaram que o consumo de FDN variou de 40,9 a 49,9g/UTM. O menor valor $(40,9 \mathrm{~g} / \mathrm{UTM})$ ocorreu 
porque o FCC foi incluído em $36 \%$ da fração concentrada da dieta e, nessa condição, o valor de extrato etéreo dietético foi superior a $6 \%$ do total de matéria seca. O farelo de castanha de caju, incluído em até $13,31 \%$ da dieta, apresenta potencial de utilização na dieta de cordeiros em terminação, caracterizando-se como importante alternativa no incremento da densidade calórica dietética. A inclusão do FCC em dietas para cordeiros de diferentes genótipos não promove alterações no consumo de nutrientes. Diferenças existentes entre genótipos influenciam o consumo de nutrientes. A inclusão de FCC promove redução sobre a digestibilidade das frações fibrosas, notadamente sobre a hemicelulose, implicando em redução da digestibilidade da MS nos cordeiros 1/2 sangue Santa Inês.

\section{REFERÊNCIAS}

ASSOCIATION OF OFFICIAL ANALYTICAL CHEMISTS - AOAC. Official methods of analysis. Washington, DC, 1995. 1015 p.

BARROS, N.N.; VASCONCELOS, V.R.; ARAÚJO, M.R.A.; MARTINS, E.C. Influência do grupo genético e da alimentação sobre o desempenho de cordeiros em confinamento. Pesquisa Agropecuária Brasileira, v.38, n.9, p.1111-1116, 2003.

CUNHA, M.G.G.; CARVALHO, F.F.R.; VÉRAS, A.S.C.; BATISTA, A.M.V. Desempenho digestibilidade aparente em ovinos confinados alimentados com dietas contendo níveis crescentes de caroço de algodão integral. Revista Brasileira de Zootecnia, v.37, n.6, p.1103-1111, 2008.
LOPES, N.C.M.; JAEGER, S.M.P.L.; OLIVEIRA, R.L.; OLIVEIRA, G.J.C.; MELO JUNIOR, A.S.; FARIA, M.M. S.; STRADA, E.S.O.; SILVA, A.M. Peso, rendimento e cortes da carcaça de cordeiros recebendo dieta a base de palma forrageira (Opuntia ficus-indica, Mill) amonizada. Magistra, v.20, p.140-145, 2008.

MACEDO JÚNIOR, G.L.; PÉREZ, J.R.O.; ALMEIDA, T.R.V.; PAULA, O.J.; FRANÇA, P.M.; ASSIS, R.M. Influência de diferentes níveis de FDN dietético no consumo e digestibilidade aparente de ovelhas Santa Inês. Ciência Agrotécnica, v.30, n.3, p.547-553, 2006.

MAIA, F.J.; BRANCO, A.F.; MOURO, G. F.; CONEGLIAN, S.M.; SANTOS, G.T.; MINELLA, T.F.; GUIMARÃES, K.C. Inclusão de fontes de óleo na dieta de cabras em lactação: Produção, composição e perfil dos ácidos graxos do leite. Revista Brasileira de Zootecnia, v.35, p.1504-1513, 2006.

MARQUES, A.V.M.S.; COSTA, R.G.; SILVA, A.M.A.; PEREIRA FILHO, J.M.; MADRUGA, M.S.; LIRA FILHO, G.E. Rendimento, composição tecidual e musculosidade da carcaça de cordeiros Santa Inês alimentados com diferentes níveis de feno de flor de seda na dieta. Revista Brasileira de Zootecnia, v.36, p.610-617, 2007.

NASCIMENTO, E.N.; ROGÉRIO, M.C.P.; BATISTA, A.S.M.; CARNEIRO, M.S.S.; VASCONCELOS, A.M.; LEITE, E.R.; LANDIM, A.V.; SILVA, V.L.; COSTA, J.B.; COSTA, H.H.A. Consumo de nutrientes e aspectos quantitativos de carcaça de ovinos em terminação alimentados com dietas contendo farelo de castanha de caju. Revista Brasileira de Saúde e Produção Animal [online], v.13, p.1099-1111, 2012. 
NATIONAL RESEARCH COUNCIL NRC. Nutrient requirements of sheep. New York: National Academy Press, 1985. 99p.

\section{NATIONAL RESEARCH COUNCIL -} NRC. Nutrient requeriments of small ruminants. Washington: National Academy Press, 2007. 362p.

PEREIRA, O.G.; SOUZA, V.G.; VALADARES FILHO, S.C.; PEREIRA, D.H.; RIBEIRO, K.G.; CECON, P.R. consumo e digestibilidade dos nutrientes e desempenho de bovinos de corte recebendo dietas com diferentes níveis de uréia. Ciência Animal Brasileira, v.9, n.3, p.552-562, 2008.

PIMENTEL, P.G.; MOURA, A.A.; NEIVA, J.N.M.; ARAÚJO, A.A.; TAIR, R.F.L. Consumo, produção de leite e estresse térmico em vacas da raça Pardo-Suíça alimentadas com castanha de caju. Arquivo Brasileiro de Medicina Veterinária e Zootecnia, v.59, p.1523-1530, 2007.

PIMENTEL, P.G.; PEREIRA, E.S.; QUEIROZ, A.C.; MIZUBUTI, I.Y.; REGADAS FILHO, J.G.L.; MAIA, I.S.G. Intake, apparent nutrient digestibility and ingestive behavior of sheep fed cashew nut meal. Revista Brasileira de Zootecnia, v.40, n.5, p.1128-1133, 2011.

RODRIGUES, M.M.; NEIVA, J.N.M.; VASCONCELOS, V.R.; LOBO, R.N.B.; PIMENTEL, J.C.M.; MOURA, A.A.A.N. Utilização do farelo de castanha de caju na terminação de ovinos em confinamento. Revista Brasileira de Zootecnia, v.32, n.1, p.240-248, 2003.
SANTOS, V.C.; EZEQUIEL, J.M.B.; OLIVEIRA, P.S.N.; GALATI, R.L.; BARBOSA, J.C. Consumo e digestibilidade em ovinos alimentados com grãos e subprodutos da canola.

Revista Brasileira de Saúde e Produção Animal [online], v.10, n.1, p.96-105, 2009.

SILVA, M.M.C.; RODRIGUES, M.T.; RODRIGUES, C.A.F.; BRANCO, R.H.; LEÃO, M.I.; MAGALHÃES, A.C.M.; MATOS, R.S. Efeito da suplementação de lipídeos sobre a digestibilidade e os parâmetros da fermentação ruminal em cabras leiteiras. Revista Brasileira de Zootecnia, v.36, n.1, p.2 46-256, 2007.

SILVA, V.L.; ROGÉRIO, M.C.P.; ALVES, A.A.; BOMFIM, M.A.D.; LANDIM, A.V.; LEITE, E.R.; COSTA, H.H.A.; FREIRE, A.P.A.

Comportamento ingestivo de cordeiros alimentados com dietas contendo farelo de castanha de caju. Revista da Faculdade de Zootecnia, Veterinária e Agronomia, v.18, p.1-17, 2011.

SNIFFEN, C.J.; O'CONNOR, J.D.; VAN SOEST, P.J.; FOX, D.J.;

RUSSELL, J.B. A net carbohydrate and protein system for evaluating cattle diets: II. Carbohydrate and protein availability. Journal of Animal Science, v.70, p.3562-3577, 1992.

TEIXEIRA, D.B.; BORGES, I. Efeito do nível de caroço de algodão sobre o consumo e digestibilidade da fração fibrosa do feno de braquiária decumbens em ovinos . Arquivo Brasileiro de Medicina Veterinária e Zootecnia, v.57,p.229-233, 2005.

UNIVERSIDADE FEDERAL DE VIÇOSA - UFV. Sistema para análise estatística e genética- SAEG. Versão 
8.0. Viçosa, MG: Fundação Arthur

Bernardes, 2001. 150p.

Van SOEST, P.J. Nutritional ecology of the ruminant. $2^{\text {th }}$. Ithaca, New York: Cornell University Press, 1994. 476p.

Van SOEST, P.J.; ROBERTSON, J.B.; LEWIS, B.A. Methods for dietary fiber, neutral detergent fiber, and nonstarch polysaccharides in relation to animal nutrition. Journal of Dairy Science, v.74, n.10, p.3583-3597, 1991.
YAMAMOTO, S.M.; SILVA

SOBRINHO, A.G.; VIDOTTI, R.M.;

HOMEM JUNIOR, A.C.; PINHEIRO,

R.S.B.; Buzzulini, C. Desempenho e digestibilidade dos nutrientes em cordeiros alimentados com dietas contendo silagem de resíduos de peixe. Revista Brasileira de Zootecnia, v.36, n.4, p.1131-1139, 2007.

Data de recebimento: 16/05/2013

Data de aprovação: 04/12/2013 